\title{
miR-1269b Drives Cisplatin Resistance of Human Non-Small Cell Lung Cancer via Modulating the PTEN/PI3K/AKT Signaling Pathway
}

This article was published in the following Dove Press journal: OncoTargets and Therapy

\author{
Wu Yang' \\ Wei Xiao ${ }^{2}$ \\ Zhengrong $\mathrm{Cai}^{3}$ \\ Shidai Jin' \\ Tian $\mathrm{Li}^{3}$ \\ 'Department of Oncology, The First \\ Affiliated Hospital of Nanjing Medical \\ University, Nanjing, Jiangsu Province \\ 210029, People's Republic of China; \\ ${ }^{2}$ Department of Radiotherapy, Nanjing \\ Chest Hospital, Nanjing, Jiangsu Province \\ 210029, People's Republic of China; \\ ${ }^{3}$ Department of Respiratory Medicine, \\ Nanjing Chest Hospital, Nanjing, Jiangsu \\ Province 210029, People's Republic of \\ China
}

Correspondence: Shidai Jin Department of Oncology, The First Affiliated Hospital of Nanjing Medical University, No. 300 Guangzhou Road, Nanjing, Jiangsu Province 21 0029, People's Republic of China

Email zqoipfi6392638@126.com

Tian Li

Department of Respiratory Medicine, Nanjing Chest Hospital, No. 215

Guangzhou Road, Nanjing, Jiangsu Province

210029 , People's Republic of China

Email jqcmfzr93987384@I26.com
Background: MiRNAs have been reported to induce certain drug resistance in multiple solid tumors via various mechanisms. Our study aimed to investigate whether miRNA-1269b was involved in the chemoresistance and the progression of non-small cell lung cancer (NSCLC).

Methods: MTT and colony formation assay were conducted to determine cell proliferation and cell apoptosis was analyzed by flow cytometry with annexin V/PI. Luciferase reporter assay was performed to validate miRNA-targeting sequences. The function of miR-1269b in cisplatin-resistant was evaluated in vivo in a mouse tumor model.

Results: We found that miR-1269b expression was up-regulated in cisplatin-resistant NSCLC specimens and NSCLC cell lines, which resulted in the promotion of chemoresistance and tumorigenicity. miR-1269b overexpression enhanced drug resistance and promoted cell proliferation in vitro and tumor growth in vivo, with reduced apoptosis rate of A549 cells inin vitro cell culture. Mechanistically, we identified PTEN as the direct target of miR-1269b, and the PTEN level was negatively correlated with miR-1269b in NSCLC specimens. Further study demonstrated that miR-1269b targeted PTEN to modulate PI3K/AKT signaling pathway.

Conclusion: In conclusion, these findings suggest that the miR-1269b/PTEN/PI3K/AKTmediated network might promote cisplatin resistance in NSCLC, and that miR-1269b can be a potential therapeutic target for chemoresistance in NSCLC patients.

Keywords: miR-1269b, NSCLC, cisplatin resistant, PTEN, PI3K/AKT signaling

\section{Background}

Non-small cell lung cancer (NSCLC) is the most malignant lung cancer and the primary cause of cancer-related death worldwide. ${ }^{23}$ Although various improvements in early diagnosis and novel targeted therapies displayed promising results, most NSCLC patients are diagnosed at advanced stage and patients still have poor five-year survival rates. ${ }^{6}$ Thus, it is critically important to identify novel diagnostic biomarkers and develop efficient therapeutic strategies for NSCLC.

Cisplatin is one of the most commonly used chemo drugs for NSCLC. ${ }^{19}$ However, chemotherapy using cisplatin is limited by the occurrence of drug resistance. ${ }^{8}$ The underlying mechanisms of cisplatin-resistant seem to be complicated and multiple signaling pathways were involved. ${ }^{9,13,25}$ Multiple signaling pathways have been reported to be involved in cisplatin chemoresistance of NSCLC including JAK2/STAT3 signaling, Wnt signaling, AKT/mTOR signaling and MDM2/p53 signaling pathway. ${ }^{21,22,24,28}$

MicroRNAs (miRNAs) participate in many biological procedures like cell cycles, proliferation, apoptosis and metastasis. ${ }^{26}$ Recent work has demonstrated that miRNA 
expression profiles were correlated with the characteristic parameters in different cancers, which could be used for cancer diagnosis. ${ }^{16}$ Increasing evidence showed that miRNAs were novel biomarkers and promising therapeutic targets for NSCLC diagnosis and therapy. ${ }^{17}$ Interestingly, more investigations proved that miRNAs also mediated the cisplatin-resistance of NSCLC. For instances, Yong et al found that high mobility group A 2 was a novel target of miR-26a and decreasing miR-26a level led to cisplatin resistance in NSCLC by regulating AKT signaling, ${ }^{30}$ miRNA-17 family (miR-17, 20a, 20b) regulated cisplatin-resistant by targeting TGF $\beta$ R2 of TGF $\beta$ signaling in NSCLC; ${ }^{11}$ miR1244 managed cisplatin-resistance of NSCLC by targeting TP53 regulated signaling pathways. ${ }^{15}$

MiR-1269b, which belongs to the miR-1269 family, promoted metastasis in colorectal cancer, hepatocellular carcinoma and lung cancer. ${ }^{2,5,29}$ Here, we studied whether miR-1269b was also involved in the cisplatin-resistance of NSCLC. Overexpression of miR-1269b promoted cell proliferation in vitro and tumor growth in vivo and enhanced drug resistance, with reduced apoptosis rate of A549 cells in vitro cell culture. We further identified PTEN as the direct target of miR-1269b and miR-1269b targeted PTEN to modulate the activation of $\mathrm{PI} 3 \mathrm{~K} / \mathrm{AKT}$ signaling and promote cisplatin resistance in NSCLC. The results indicated that miR-1269b could be a target for chemoresistance in NSCLC patients.

\section{Methods}

\section{Clinical Samples and Cell Lines}

The study was approved by The First Affiliated Hospital of Nanjing Medical University. All patients signed informed consent. Lung tissue samples were obtained from the resected lungs of NSCLC patients between 1997 and 2010. A total of 32 tumor samples with adjacent normal tissues were selected according to the clinical database and stored at $-80{ }^{\circ} \mathrm{C}$ for the following test.

HA549, SPCA1, H1299, H358, PC9, A549/DDP and $16 \mathrm{HBE}$ were obtained from the Shanghai Academy of Sciences (Shanghai, China). They were cultured in RPMI-1640 medium with 10\% FBS, $100 \mathrm{U} / \mathrm{mL}$ penicillin and $100 \mu \mathrm{g} / \mathrm{mL}$ streptomycin (Gibco, New York, USA) in $5 \% \mathrm{CO}_{2}$ cell incubator at $37{ }^{\circ} \mathrm{C}$.

\section{Chemotherapeutic Response}

The chemotherapeutic response was assessed as previously described. ${ }^{7}$ Briefly, subjects were defined into responders and nonresponders. Cisplatin chemotherapy was performed for 4 or more cycles.

\section{Real-Time qPCR}

According to the manufacturer's instructions, TRIzol reagent (Invitrogen, CA, USA) was used to isolate the total RNA from tissues and cells. Then, cDNA was generated using total RNA and the PrimeScript RT reagent (Takara, Kusatsu, Japan), and qRT-PCR was performed with SYBR Green Master Mix II (Takara) on an ABI 7900 fast real-time PCR system (ABI, CA, USA). The expression levels of mRNA and miRNA were normalized using GAPDH and small RNA RNU6B (U6) as endogenous controls. The $2^{-\Delta \Delta C T}$ method was used to quantify the relative levels of miR-1269b and PTEN mRNA. Each sample was run in triplicate.

\section{Plasmid Transfection}

The lentiviral miRNA and inhibitors (LV-hsa-miR-1269bmimics, miR-NC, LV-hsa-miR-1269b-inhibitor, and LV-NCinhibitor), plasmid PTEN, and control vector were obtained from Gene Pharma (Shanghai, China). Transfection using lipofectamine 3000 reagent (Invitrogen) was performed when the cells reached $80 \%$ confluence.

\section{Cell Proliferation and Colony Formation}

MTT assay was utilized to examine cell proliferation, and $3 \times 10^{3}$ cells/well were seeded. After treated with the indicated drug combinations for 2 days, the absorbance at 570 nm was measured using Envision (PerkinElmer, Norwalk, CT). The IC50 (concentration of drug needed to inhibit cell growth by $50 \%$ ) is generated from the dose-response curves for each cell line. For colony formation assay, 400 cells/well were seeded in a 6-well plate and cultured for 14 days. Cells were fixed and stained with crystal violet solution (Sigma, MO, USA). The colonies containing more than 50 cells were counted under a microscope.

\section{Apoptosis Analysis}

Cells transfected with miR-NC, miR-1269b, NC-inhibitor or miR-1269b inhibitor were treated with or without cisplatin for $48 \mathrm{hrs}$, apoptosis rate was analyzed by flow cytometry with annexin V/PI double staining kit (BD, San Jose, CA).

\section{Luciferase Reporter Assay}

The 3'-Untranslated region (3'-UTR) of PTEN or the targeting site containing mutated sequence was magnified and 
implanted into the downstream of the luciferase reporter gene in the pmir-GLO-promoter vector (Promega, USA), named PTEN-3'-UTR-WT and PTEN-3'-UTR-MUT. The mutant 3' UTR of PTEN was magnified by applying wild PTEN 3'UTR as the template, and the mutant plasmid was obtained through the Site-Directed Mutagenesis Kit. A549 transfected with PTEN-3'-UTR-WT or PTEN-3'-UTRMUT, together with miR-1269b mimics or NC control with Lipofectamine 2000 (Invitrogen). And Renilla luciferase pRL-TK vector was used as a control. After being transfected 2 days, cells were lysed with RIPA buffer. Luciferase activity was evaluated consequently via DualLuciferase Assay System (Promega) following the producer's manual.

\section{In vivo Study}

Female BALB/C mice (5-6 weeks) were purchased from The First Affiliated Hospital of Nanjing Medical University and Nanjing Chest Hospital. Xenograft experiments were approved by the Ethics board of The First Affiliated Hospital of Nanjing Medical University and Nanjing Chest Hospital. The A549 cells that stably overexpressed miR$1269 \mathrm{~b}$ or downregulated miR-1269b were harvested. A total of $4 \times 10^{6}$ cells (A549 or A549/DDP) were implanted into the side of the mouse. Cisplatin was administrated intraperitoneally twice a week at the dose of $3 \mathrm{mg} / \mathrm{kg}$. After 4 weeks, the development of the tumor was calculated with the formula: Volume $=1 / 2 \times$ length $\times$ width $^{2}$.

\section{Immunohistochemical (IHC) Analysis}

Patient samples were fixed using $4 \%$ formalin and embedded in paraffin. Paraffin sections were dewaxed, rehydrated and incubated with anti-PTEN (Abcam, Cambridge, England) overnight at $4{ }^{\circ} \mathrm{C}$ after blocking. Then, the sections were washed 3 times with PBS and incubated with HRP-polymer conjugated secondary antibody. The sections were stained and observed.

\section{Western Blotting Analysis}

Cells were lysed in RIPA buffer (Beyotime, Shanghai, China) containing protease inhibitor cocktail (SigmaAldrich, MO, USA). Western blotting was conducted with primary antibodies: PTEN (Abcam), PI3K, AKT, p-AKT and GAPDH (all from Cell Signaling Technology).

\section{Statistical Analysis}

Data were presented as the means \pm standard deviation of 3 independent experiments. The results were analyzed using
Student's $t$-test where necessary. Pearson's correlation was estimated between miR-1269b and PTEN. Graphpad Prism 6.0 was used to perform statistical analysis and $P$ values $<0.05$ were considered statistically significant.

\section{Results}

High Expression of miR-1269b in NSCLC Is Associated with Chemoresistance

The miR-1269b expression level was analyzed in primary NSCLC tumor and adjacent normal tissues. Figure 1A shows that miR-1269b expression was significantly higher in both responder tumors and non-responder tumors compared with that in corresponding control normal tissues. Moreover, miR$1269 \mathrm{~b}$ level in non-responder tumors was remarkably higher than that in responder tumors (Figure 1A). The overall survival of NSCLC patients with high miR-1269b suggested miR$1269 \mathrm{~b}$ as favorable cancer prognostic markers (Figure 1B).

MiR-1269b expression was also evaluated in a panel of NSCLC cells (A549, H1299, SPCA1, H358 and PC9), which was significantly higher compared with that in human bronchial epithelial cells (16HBE) (Figure 1C). Moreover, the expression of miR-1269b in drug-resistant cell line A549/ cisplatin (A549/DDP) was drastically higher than that in the parental lung cancer cell line A549 (Figure 1C). In addition, we found that paclitaxel (PTX; 100nM for $72 \mathrm{~h}$ ) treatment also significantly increased miR-1269b expression in A549 cells (Fig. S1A). Thus, we next investigated whether miR-1269b regulated chemoresistance in NSCLC. A549 and A549/DDP cells were transfected with miR-1269b mimics or miR-1269b inhibitor to up-regulate or down-regulate miR-1269b. The transfection of miR-1269b mimics significantly increased miR-1269b expression ( $\mathrm{P}<0.001$; Fig. S1B), while miR$1269 \mathrm{~b}$ inhibitor significantly reduced its expression in both A549 and A549/DDP cells ( $\mathrm{P}<0.001$; Fig. S1B). Moreover, miR-1269b overexpression increased the cell viability, while miR-1269b knockdown inhibited the cell viability of A549 and A549/DDP cells (Fig. S1C). As shown in Figure 1D, miR-1269b overexpression with miR-1269b mimics caused a significant resistance to DDP, while decreased expression of miR-1269b with miR-1269b inhibitor transfection sensitized the drug response of A549 cell line.

\section{MiR-I 269b Enhances the Chemoresistance of NSCLC Cells}

To test the potential effects of miR-1269b on chemoresistance, both parental A549 cells and cisplatin-resistant A549/ DDP cells were transfected with miR-NC/miR-1269b 

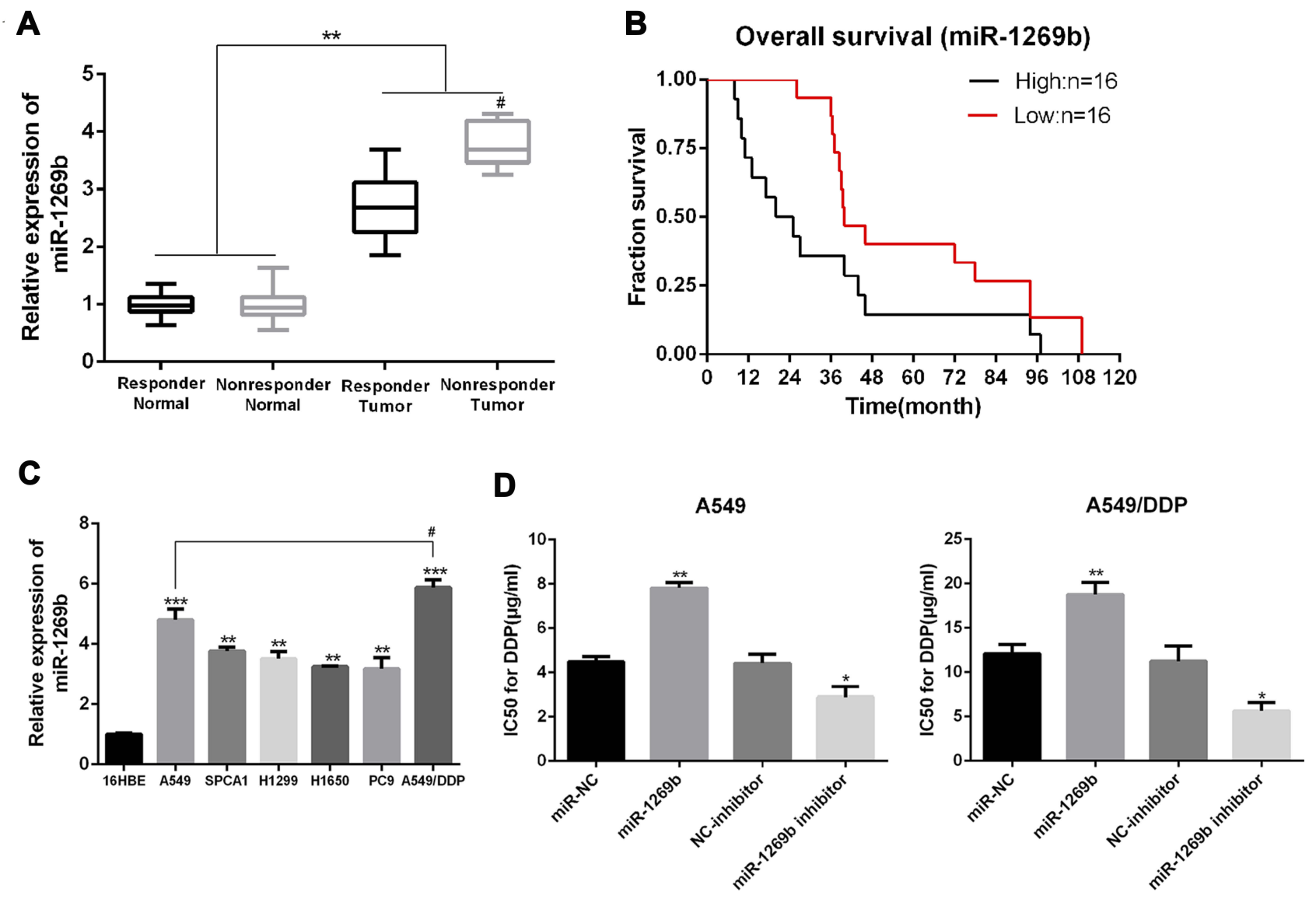

Figure I MiR-1269b is associated with chemoresistance and survival in NSCLC. (A) real-time qPCR analysis of the expression levels of miR-I269b in NSCLC tumor tissues and adjacent normal tissues from the responder group $(n=16)$ and nonresponder group $(n=16)$. (B) Overall survival Kaplan-Meier curves were based on miR-I269b expression, median miRNA expression was used to define low and high expression. (C) real-time qPCR analysis of miR-I269b in human NSCLC cell lines. (D) IC50 of DDP in NSCLC cells transfected with miRNA-197 inhibitor or mimics was examined by MTT assay. ${ }^{*} p<0.05$; ${ }^{* *} p<0.01$; ${ }^{* * *} \mathrm{p}<0.00 \mathrm{I}$ compared to relative control. ${ }^{*} \mathrm{P}<0.05$ compared to nonresponder normal group.

Abbreviations: DDP, cisplatin; NC, negative control.

mimics or NC-inhibitor/miR-1269b inhibitor. Colony formation assay showed that the proliferation ability of A549 cells was significantly enhanced by the transfection of miR-1269b mimics, while the introduction of miR-1269b inhibitor obviously inhibited the proliferative ability of A549/DDP cells (Figure 2A and B). Figure $2 \mathrm{C}$ and D displayed that a decrease of apoptotic rate induced by miR-1269b mimics in A549 cells, and an increase of apoptotic rate induced by miR1269b inhibitor in A549/DDP cells (Figure 2C and D). Therefore, we demonstrated that miR-1269b could enhance the chemoresistance of NSCLC cells.

\section{MiR- I 269b Directly Targets PTEN in NSCLC Cells}

We performed bioinformatics analysis using database Starbase. It was speculated that PTEN might contain a putative binding site with miR-1269b (Figure 3A). Luciferase reporter vectors with WT PTEN 3' UTR sequences (PTEN-3'-UTR-WT) or mutated miR-1269b binding sites (PTEN-3'-UTR-MUT) were constructed and co-transfected with miR-NC or miR-1269b mimics into A549 cells. Figure 3B shows a significant decrease in relative luciferase activity in PTEN-3'-UTR-WT and miR-1269b mimics group. Consistent with the high expression of miR-1269b in NSCLC (Figure 1A), PTEN expression was significantly lower in NSCLC tissues than that in matched normal tissues (Figure 3C and D). In addition, PTEN mRNA and protein were inhibited by miR-1269b mimics but elevated by miR-1269b inhibitor in A549 cells (Figure 3E). Moreover, a negative correlation was found between miR-1269b and PTEN mRNA (Figure 3F). The above indicated that miR-1269b might directly target PTEN in NSCLC cells. 

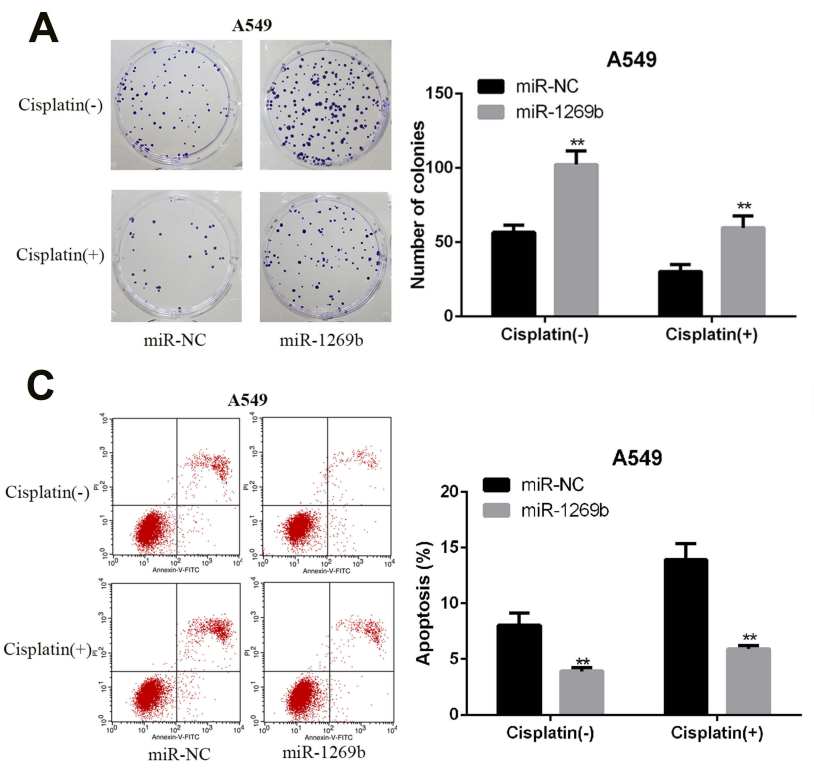
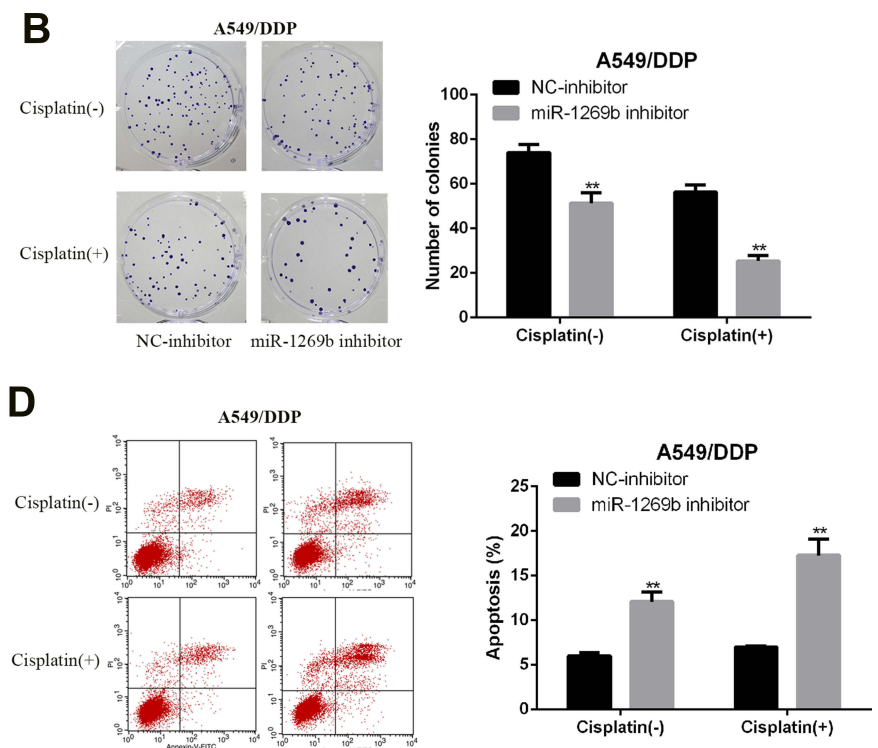

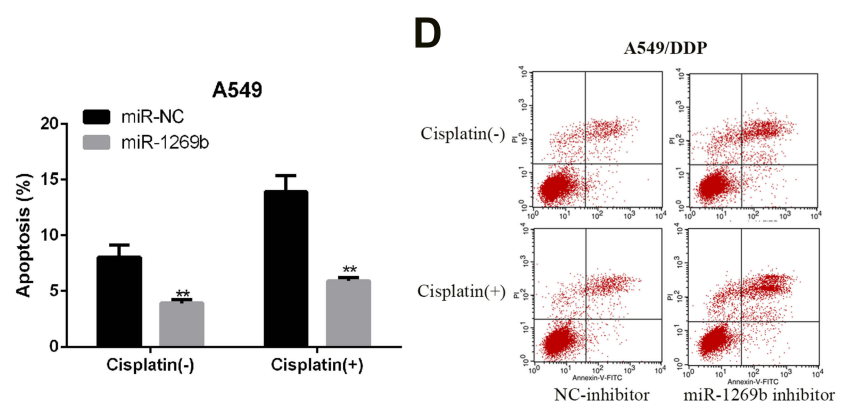

Figure 2 MiR-I269b enhances the chemoresistance of NSCLC cells. (A) Colony formation assay was used to examine the proliferation ability of A549 cells treated with or without DPP $(2 \mu \mathrm{g} / \mathrm{mL})$ after miR-NC/miR-1269b transfection. (B) Colony formation assay was used to measure the proliferation ability of A549/DDP cells treated with or without DPP $(5 \mu \mathrm{g} / \mathrm{mL})$ after NC-inhibitor/miR-1269b inhibitor transfection. (C) Flow cytometry analysis of the apoptotic rate in cisplatin-treated A549 cells after miR-NC/miR-1269b transfection. (D) Flow cytometry analysis of the apoptotic rate in cisplatin-treated A549/DDP cells after NC-inhibitor/miR-I269b inhibitor transfection. $*^{*} p<0.01$. (-), without cisplatin treatment $(+)$ treated with DPP.

\section{MiR-I269b Contributes to Chemoresistance of NSCLC Cells via PTEN/PI3K/AKT Signaling Pathway}

MiR-1269b mimics resulted in enhanced IC50 values of DDP in A549 cells, which was significantly reversed by miR-1269b mimics/PTEN cotransfection (Figure 4A, left panel). The reduction of IC50 values by miR-1269b inhibitor transfection in A549/DDP cells was further enhanced by PTEN overexpression (Figure 4A, right panel). Moreover, the effects of miR-1269b on colony formation capacity of A549 cells were also partially reversed due to the transfection of PTEN overexpression vector, while the colony formation capacity of A549/DDP cells was further inhibited by miR-1269b inhibitor/PTEN cotransfection compared with that of miR-1269b inhibitor transfection only (Figure 4B). Consistently, overexpression of PTEN remarkably reversed miR-1269b-induced anti-apoptotic apoptosis of A549 cells and further enhanced miR-1269b inhibitor-induced apoptosis of A549/DDP cells (Figure 4C). To explore how miR-1269b/PTEN axis regulated chemosensitivity, cell apoptosis and proliferation, we examined the activation of the PTEN-regulated PI3K/AKT signaling pathway. As showed in Figure 4D, miR-1269b mimics increased $\mathrm{PI} 3 \mathrm{~K}$ expression and $\mathrm{p}$-AKT/AKT ratio, while PTEN overexpression reversed its effects. In contrast, miR$1269 \mathrm{~b}$ inhibitor reduced PI3K expression and p-AKT/AKT ratio, which was further inhibited by PTEN overexpression in
A549/DDP cells. In summary, our data elucidated that miR$1269 \mathrm{~b}$ might contribute to the chemosensitivity of NSCLC cells via modulating PTEN/PI3K/AKT pathway.

\section{Targeting miR-1269b Overcomes \\ Cisplatin Resistance in vivo}

To test whether miR-1269b participates in cisplatin resistance internally, A549 cells or A549/DDP cells with miR-1269b mimics or inhibitor were implanted in mice. As shown in Figure 5A, miR-1269b/A549 cells group had larger sizes of tumors than the miR-NC/A549 group. However, the average size of the tumor in the miR-1269b inhibitor+A549/DDP group was significantly smaller than that in the $\mathrm{NC}$-inhibitor +A549/DDP group (Figure 5B). We further tested the PTEN/ $\mathrm{PI} 3 \mathrm{~K} / \mathrm{AKT}$ signaling. Western blot results revealed that miR1269b mimics significantly inhibited PTEN expression and increased PI3K and p-AKT expression in A549 cells with cisplatin, while miR-1269b inhibitor significantly increased PTEN expression, inhibited PI3K and p-AKT expression in A549/DDP cells with cisplatin (Figure 5C and D). The results suggested that manipulating miR-1239b might abolish cisplatin resistance by targeting PTEN/PI3K/AKT pathway.

\section{Discussion}

In this study, we found that miR-1269b was significantly up-regulated in NSCLC patients and was associated with 
A

wt-PTEN

3'-AAUUAAUUCCACAGUGUUAC-5'

hsa-miR-1269b 5'-CCAGUCCUGAUGUCACAAUC-3'

mut-PTEN 3'-AAUUAAUUCCUAACACCCUU-5'

C

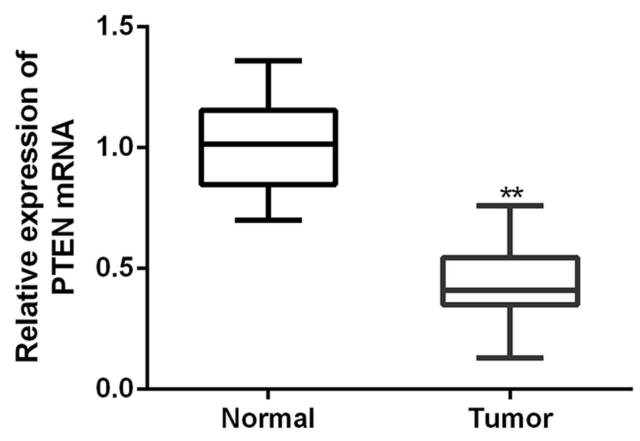

E
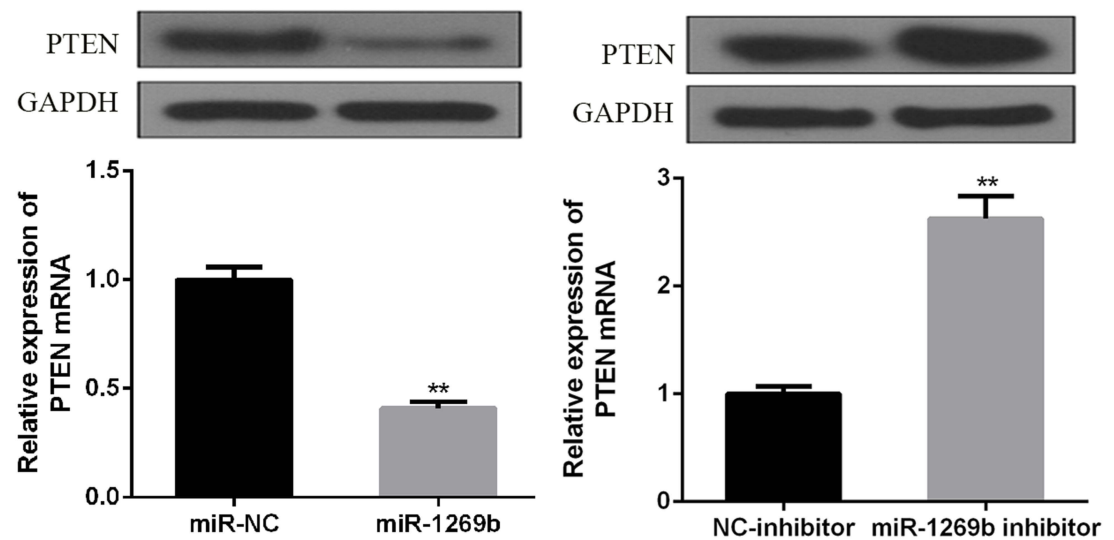

$\mathbf{F}$

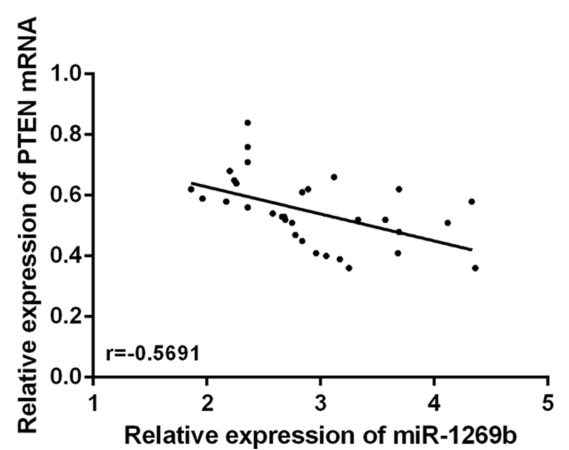

Figure 3 MiR-1269b directly targets PTEN in NSCLC. (A) Bioinformatics analysis showed the predicted binding site between miR-I269b and PTEN. (B) The relative luciferase activity of A549 cells was detected after PTEN-3'-UTR-WT or PTEN-3'-UTR-MUT co-transfected with miR-I269b mimic or miR-NC. (C, D) The expression of PTEN mRNA and protein in NSCLC tissues and matched normal tissues was detected by using qRT-PCR ( $n=32$ ) and IHC, respectively. (E) The expression of PTEN mRNA and protein were analyzed in the presence of miR-1269b or miR-1269b inhibitor in A549 cells. (F) Negative correlation between miR-1269b and PTEN in NSCLC tissues $(n=32) .(* * p<0.01)$.

chemoresistance and survival. Using NSCLC cell lines, we further demonstrated that miR-1269b promoted cell proliferation and inhibited cell apoptosis, leading to the chemoresistance of NSCLC cells. Mechanistically, we found that miR-1269b targeted PTNE and modulated PI3K/AKT signaling. This is the first study reporting miR-1269b regulated drug-resistance to cisplatin in NSCLC cells.
Various miRNAs are investigated in NSCLC, which could be served as diagnostic biomarkers and therapeutic targets. ${ }^{17}$ Here we focused on miR-1269b, which has been reported to be an oncogene in hepatoma cells. ${ }^{29} \mathrm{NF}-\mathrm{\kappa B}$ activated the transcription of miR-1269b and then resulted in promoting HCC cell cycle, proliferation and migration. ${ }^{12}$ CDC40 was proved to be a target of miR-1269b, promoting 

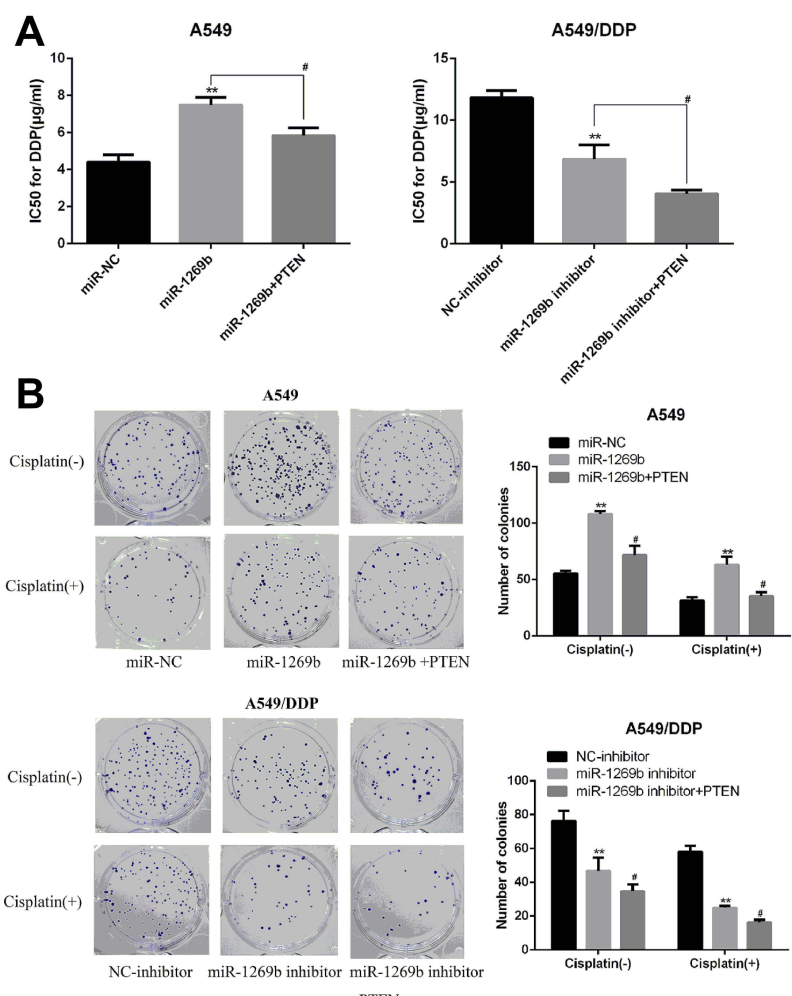

+ PTEN
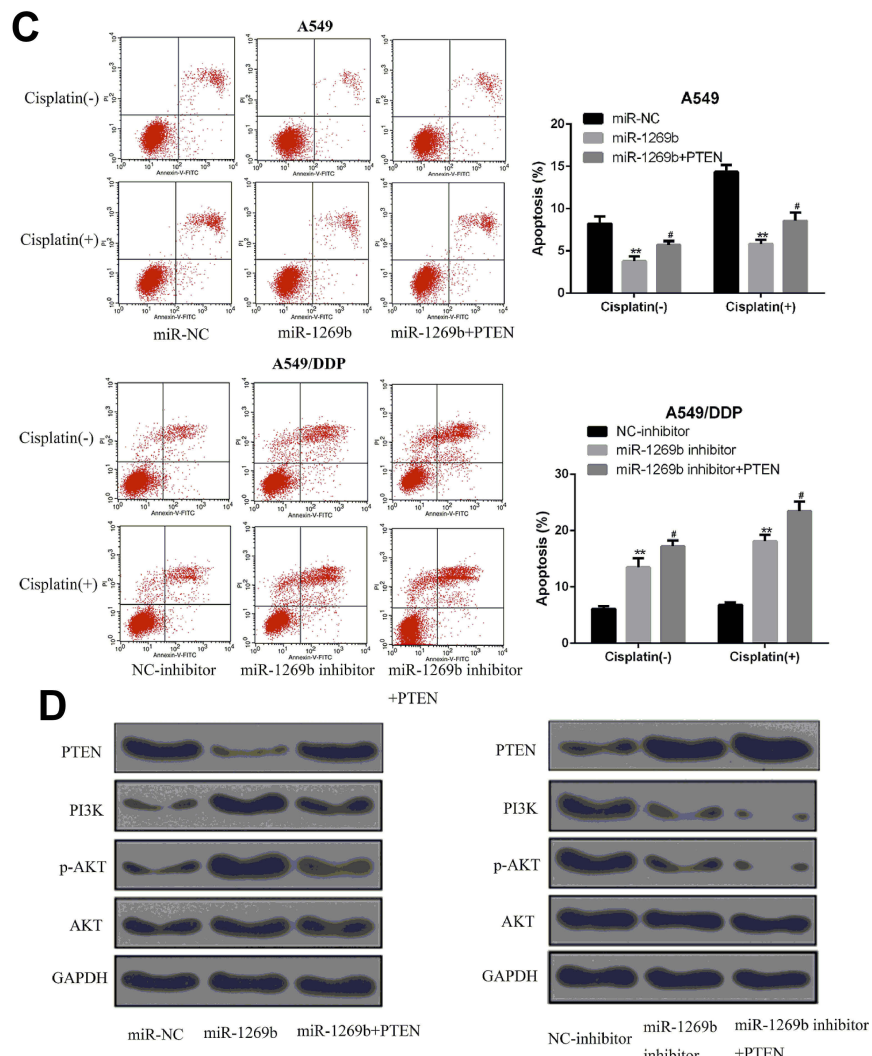

Figure 4 miR-I269b enhances the chemosensitivity of NSCLC cells via modulating PTEN/PI3K/AKT signaling pathway. (A) IC50 of DDP in A549 cells or A549/DDP cells transfected with miRNA-1269b mimics was examined by MTT assay. (B) Colony formation assay was used to measure the proliferation ability of A549 cells or A549/DDP cells as indicated. (C) Flow cytometry analysis of the apoptotic rate of A549 cells or A549/DDP cells as indicated. (D) Expression of PTEN, PI3K, AKT and P-AKT in DDPtreated A549 cells or DDP/A549 cells was detected by Western blot. ${ }^{* *} p<0.01$ compared with control group, ${ }^{\#} p<0.05$ compared with miR-I269b group or miR-I269b inhibitor group. $(-)$, without cisplatin treatment $(+)$ treated with DPP.

malignancy of HCC. ${ }^{12}$ Another study showed that TGF- $\beta$ activated miR-1269 via Sox4 and positively feedback the TGF- $\beta$ signaling by targeting Smad7 and HOXD10. ${ }^{5}$ In lung cancer, miR-1269 might promote cell survival and proliferation by targeting TP53 and caspase-9. ${ }^{2}$ Our bioinformatics analysis identified PTEN as another novel target of miR-1269.

PTEN is a known prognostic marker and tumor suppressor in NSCLC. ${ }^{27}$ Inactivation of PTEN enhanced the invasion ability of lung cancer cells by activating the PI3K/ AKT/NFkB signaling pathway. ${ }^{1}$ Consistently, the upregulation of PTEN expression could inhibit NSCLC cell proliferation and promote cell apoptosis. ${ }^{3}$ PTEN can be targeted by multiple different miRNAs in various tumors. MiR-26a inhibited PTEN expression and promoted gliomagenesis, while miR-21 overexpression contributed to cell proliferation via targeting PTEN. ${ }^{10,20}$ Using both in vitro NSCLC cell lines and in vivo mouse tumor model, we also confirmed that miR-1269b/PTEN axis regulated NSCLC chemosensitivity and cell proliferation.
Furthermore, we also found that cisplatin could increase miR-1269b expression. It is well known that miRNAs can regulate genes involved in multiple pathways such as cell death, cell proliferation, stress resistance and fat metabolism in cancer. ${ }^{18}$ Therefore, the aberrant expression of microRNA has close relationships with anticancer drug resistance phenotype. It has been well documented that transcriptional deregulation, epigenetic alterations, mutations, DNA copy number abnormalities and defective miRNA biogenesis pathway may each or together contribute to miRNA deregulation in anticancer drug resistance. ${ }^{31}$ For example, knockdown of the main miRNA processing enzyme (Dicer) results in G1 arrest and increased sensitivity to cisplatin. ${ }^{4}$ So, we speculated that the dysfunction of the main miRNA processing enzyme (Dicer) might be responsible for the miR-1269brelated cancer drug resistance in NSCLC, which still needs further verification in our next study.

Several limitations should be noticed and further investigated in our study. First, how miR-1269b expression is 
A

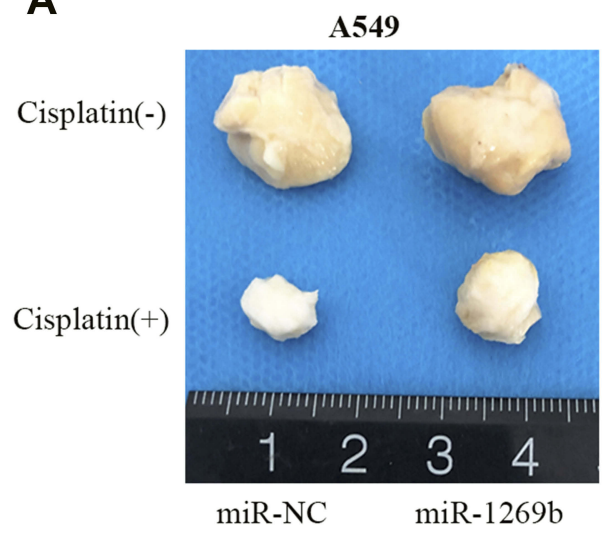

B

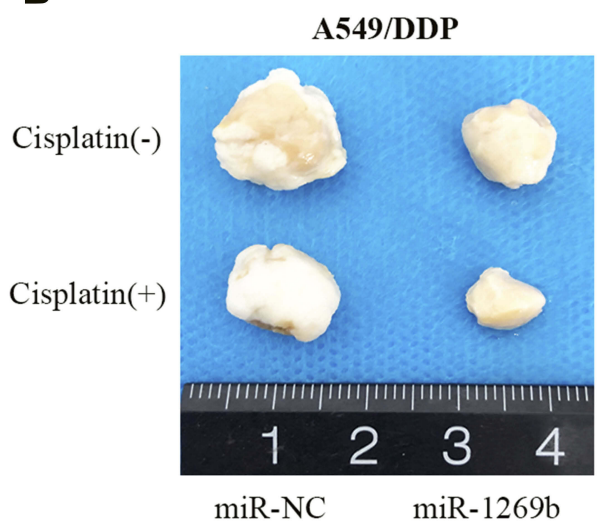

C

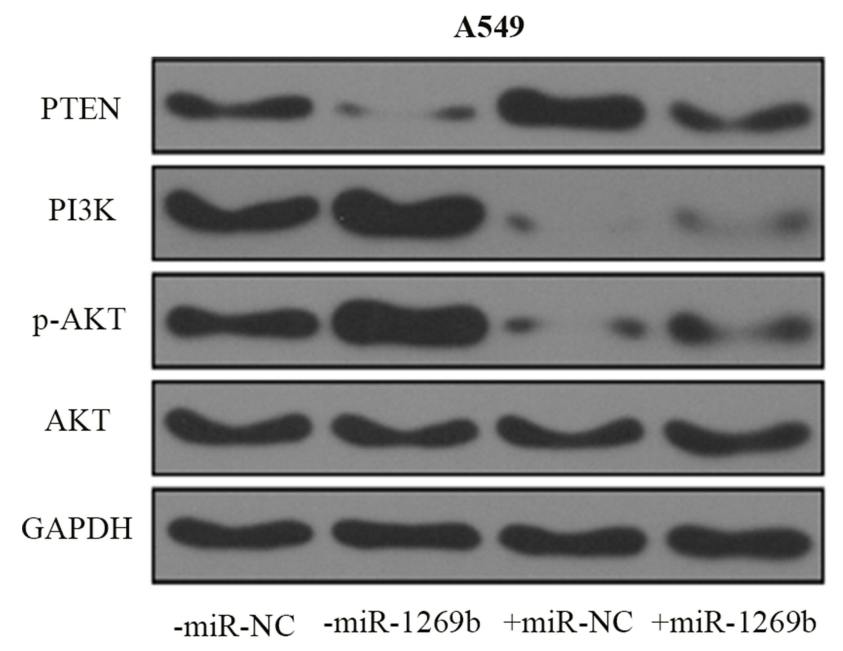

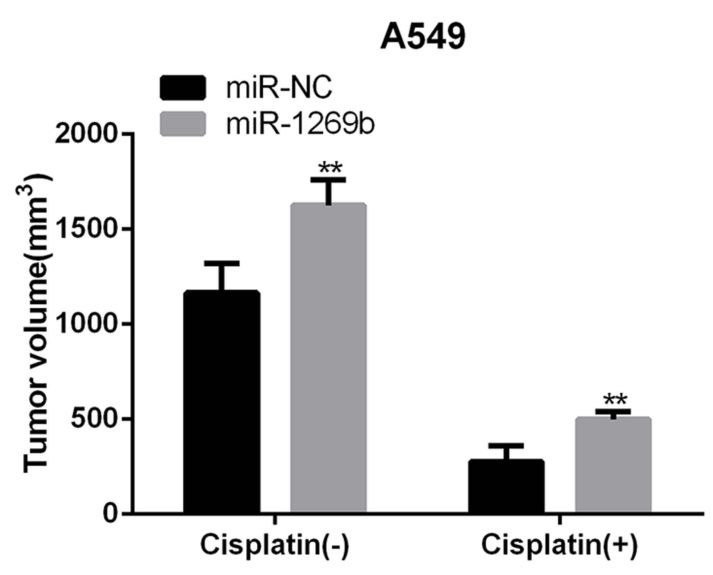

A549/DDP

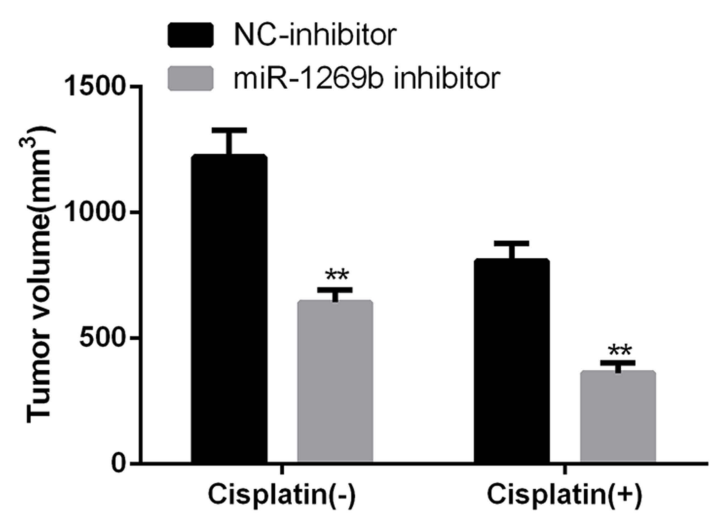

D

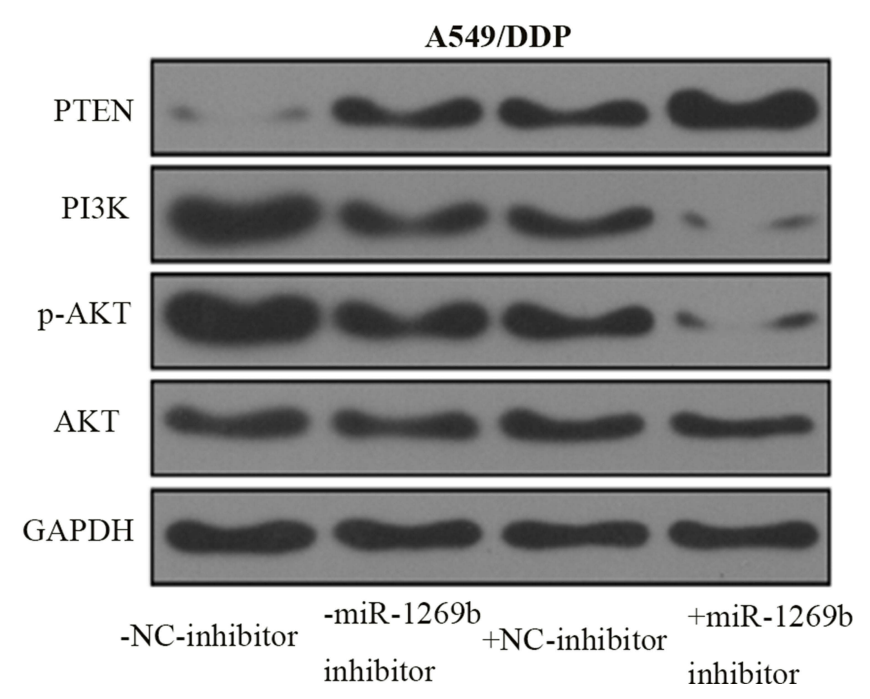

Figure 5 miR-1269b mediated cisplatin resistance in vivo. (A, B) A549 cells or A549/DDP cells with miR-1269b mimics or inhibitor were implanted into BALB/C mice. Mice were administrated with cisplatin or control buffer. Tumor volume was measured after indicated treatment for 4 weeks ( $n=6$ in each group). (C, D) Expression of PTEN, $\mathrm{PI} 3 \mathrm{~K}, \mathrm{AKT}$ and $\mathrm{p}-\mathrm{AKT}$ in tumor tissues detected by Western blot. GAPDH was used as the internal control. **p $<0.0 \mathrm{l}$ compared with control group. $(-)$, without cisplatin treatment $(+)$ treated with DPP.

regulated in NSCLC patients is still unclear. Whether there is a positive feedback mechanism between enhanced PI3K/AKT-NFKB or the NSCLC patients also have enhanced TGF- $\beta$ signaling remains unknown. ${ }^{5,12}$ Second, single miRNA can target multiple genes and single gene can be regulated by multiple miRNAs. ${ }^{14}$ Though in this 
study we demonstrated clearly that miR-1269b regulated PTEN in NSCLC, whether other miRNAs such as miR26a and miR-21 are also involved in PTEN regulation is not fully addressed. In addition, whether targeting miR$1269 \mathrm{~b}$ could be used as an efficient strategy to reverse the resistance to chemotherapy of NSCLS needs more investigation.

\section{Conclusions}

In summary, we showed that miR-1269b was overexpressed in NSCLC and miR-1269b down-regulated PTEN by targeting PTEN mRNA. MiR-1269b can modulate the activation of PI3K/AKT signaling pathway and promote cisplatin resistance in NSCLC. We found that miR-1269b could be a potential therapeutic target for chemoresistant in NSCLC patients.

\section{Data Sharing Statement}

All the data supporting our findings can be found in the "Results" section of the paper. Please contact authors for data requests.

\section{Ethics Approval}

The study has been carried out in accordance with the World Medical Association Declaration of Helsinki and its later amendments and comparable ethical standards and the protocol were approved by The First Affiliated Hospital of Nanjing Medical University. All applicable institutional guidelines for the care and use of animals were followed.

\section{Informed Consent}

Informed consent was obtained from all individual participants included in the study.

\section{Author Contributions}

All authors contributed to data analysis, drafting or revising the article, gave final approval of the version to be published, and agree to be accountable for all aspects of the work.

\section{Funding}

This research received no specific grant from any funding agency in the public, commercial, or not-for-profit sectors.

\section{Disclosure}

The authors report no conflicts of interest in this work.

\section{References}

1. Akca H, Demiray A, Tokgun O, Yokota J. Invasiveness and anchorage independent growth ability augmented by PTEN inactivation through the PI3K/AKT/NFkB pathway in lung cancer cells. Lung Cancer. 2011;73(3):302-309. doi:10.1016/j.lungcan.2011.01.012

2. Bao M, Song Y, Xia J, Li P, Liu Q, Wan Z. miR-1269 promotes cell survival and proliferation by targeting tp53 and caspase- 9 in lung cancer. Onco Targets Ther. 2018;11:1721-1732. doi:10.2147/OTT. S157715

3. Bi L, Chen J, Yuan X, Jiang Z, Chen W. Salvianolic acid A positively regulates PTEN protein level and inhibits growth of A549 lung cancer cells. Biomed Rep. 2013;1(2):213-217. doi:10.3892/br.2012.33

4. Bu Y, Lu C, Bian C, et al. Knockdown of Dicer in MCF-7 human breast carcinoma cells results in G1 arrest and increased sensitivity to cisplatin. Oncol Rep. 2009;21(1):13-17. doi:10.3892/or_00000183

5. Bu P, Wang L, Chen KY, et al. miR-1269 promotes metastasis and forms a positive feedback loop with TGF-beta. Nat Commun. 2015;6:6879. doi:10.1038/ncomms7879

6. Davies J, Patel M, Gridelli C, de Marinis F, Waterkamp D, McCusker ME. Real-world treatment patterns for patients receiving second-line and third-line treatment for advanced non-small cell lung cancer: a systematic review of recently published studies. PLoS One. 2017;12(4):e0175679. doi:10.1371/journal.pone.0175679

7. Fujita Y, Yagishita S, Hagiwara K, et al. The clinical relevance of the miR-197/CKS1B/STAT3-mediated PD-L1 network in chemoresistant non-small-cell lung cancer. Mol Ther. 2015;23(4):717-727. doi:10.1038/ mt.2015.10

8. Galluzzi L, Vitale I, Michels J, et al. Systems biology of cisplatin resistance: past, present and future. Cell Death Dis. 2014;5:e1257. doi:10.1038/cddis. 2013.428

9. Horibe S, Matsuda A, Tanahashi T, et al. Cisplatin resistance in human lung cancer cells is linked with dysregulation of cell cycle associated proteins. Life Sci. 2015;124:31-40. doi:10.1016/j. lfs.2015.01.011

10. Huse JT, Brennan C, Hambardzumyan D, et al. The PTEN-regulating microRNA miR-26a is amplified in high-grade glioma and facilitates gliomagenesis in vivo. Genes Dev. 2009;23(11):1327-1337. doi:10.1101/gad.1777409

11. Jiang Z, Yin J, Fu W, et al. MiRNA 17 family regulates cisplatin-resistant and metastasis by targeting TGFbetaR2 in NSCLC. PLoS One. 2014;9(4): e94639. doi:10.1371/journal.pone.0094639

12. Kong XX, Lv YR, Shao LP, et al. HBx-induced MiR-1269b in NF-kappaB dependent manner upregulates cell division cycle 40 homolog (CDC40) to promote proliferation and migration in hepatoma cells. $J$ Transl Med. 2016;14(1):189. doi:10.1186/s12967-016-0949-y

13. Kumar Biswas S, Huang J, Persaud S, Basu A. Down-regulation of Bcl-2 is associated with cisplatin resistance in human small cell lung cancer H69 cells. Mol Cancer Ther. 2004;3(3):327-334.

14. Lee HY, Han SS, Rhee $\mathrm{H}$, et al. Differential expression of microRNAs and their target genes in non-small-cell lung cancer. Mol Med Rep. 2015;11(3):2034-2040. doi:10.3892/mmr.2014.2890

15. Li W, Wang W, Ding M, Zheng X, Ma S, Wang X. MiR-1244 sensitizes the resistance of non-small cell lung cancer A549 cell to cisplatin. Cancer Cell Int. 2016;16:30. doi:10.1186/s12935-016-0305-6

16. Lu J, Getz G, Miska EA, et al. MicroRNA expression profiles classify human cancers. Nature. 2005;435(7043):834-838. doi:10.1038/ nature 03702

17. Lu J, Zhan Y, Feng J, Luo J, Fan S. MicroRNAs associated with therapy of non-small cell lung cancer. Int J Biol Sci. 2018;14 (4):390-397. doi:10.7150/ijbs.22243

18. Ma J, Dong C, Ji C. MicroRNA and drug resistance. Cancer Gene Ther. 2010;17(8):523-531. doi:10.1038/cgt.2010.18

19. Pillai RN, Ramalingam SS. Advances in the diagnosis and treatment of non-small cell lung cancer. Mol Cancer Ther. 2014;13(3):557-564. doi:10.1158/1535-7163.MCT-13-0669 
20. Qin X, Yan L, Zhao X, Li C, Fu Y. microRNA-21 overexpression contributes to cell proliferation by targeting PTEN in endometrioid endometrial cancer. Oncol Lett. 2012;4(6):1290-1296. doi:10.3892/ ol.2012.896

21. Stewart DJ, Chang DW, Ye Y, et al. Wnt signaling pathway pharmacogenetics in non-small cell lung cancer. Pharmacogenomics J. 2014;14(6):509-522. doi:10.1038/tpj.2014.21

22. Tang XL, Yan L, Zhu L, Jiao DM, Chen J, Chen QY. Salvianolic acid A reverses cisplatin resistance in lung cancer A549 cells by targeting c-met and attenuating Akt/mTOR pathway. J Pharmacol Sci. 2017;135(1):1-7. doi:10.1016/j.jphs.2017.06.006

23. Torre LA, Bray F, Siegel RL, Ferlay J, Lortet-Tieulent J, Jemal A. Global cancer statistics, 2012. CA Cancer J Clin. 2015;65(2):87-108. doi:10.3322/caac. 21262

24. Wang M, Lin T, Wang Y, et al. CXCL12 suppresses cisplatin-induced apoptosis through activation of JAK2/STAT3 signaling in human non-small-cell lung cancer cells. Onco Targets Ther. 2017;10:3215-3224. doi:10.2147/OTT.S133055

25. Wang M, Liu ZM, Li XC, Yao YT, Yin ZX. Activation of ERK1/2 and Akt is associated with cisplatin resistance in human lung cancer cells. J Chemother. 2013;25(3):162-169. doi:10.1179/19739 47812Y.0000000056
26. Winter J, Jung S, Keller S, Gregory RI, Diederichs S. Many roads to maturity: microRNA biogenesis pathways and their regulation. Nat Cell Biol. 2009;11(3):228-234. doi:10.1038/ncb0309-228

27. Xiao J, Hu CP, He BX, et al. PTEN expression is a prognostic marker for patients with non-small cell lung cancer: a systematic review and meta-analysis of the literature. Oncotarget. 2016;7(36):57832-57840. doi:10.18632/oncotarget.11068

28. Xing Y, Liu Y, Liu T, et al. TNFAIP8 promotes the proliferation and cisplatin chemoresistance of non-small cell lung cancer through MDM2/p53 pathway. Cell Commun Signal. 2018;16(1):43. doi:10.1186/s12964-018-0254-x

29. Yang XW, Shen GZ, Cao LQ, et al. MicroRNA-1269 promotes proliferation in human hepatocellular carcinoma via downregulation of FOXO1. BMC Cancer. 2014;14:909. doi:10.1186/14712407-14-909

30. Yang Y, Zhang P, Zhao Y, Yang J, Jiang G, Fan J. Decreased MicroRNA-26a expression causes cisplatin resistance in human non-small cell lung cancer. Cancer Biol Ther. 2016;17(5):515-525. doi:10.1080/15384047.2015.1095405

31. Zheng T, Wang J, Chen X, Liu L. Role of microRNA in anticancer drug resistance. Int $J$ Cancer. 2010;126(1):2-10. doi:10.1002/ ijc. 24782

\section{Publish your work in this journal}

OncoTargets and Therapy is an international, peer-reviewed, open access journal focusing on the pathological basis of all cancers, potential targets for therapy and treatment protocols employed to improve the management of cancer patients. The journal also focuses on the impact of management programs and new therapeutic agents and protocols on patient perspectives such as quality of life, adherence and satisfaction. The manuscript management system is completely online and includes a very quick and fair peer-review system, which is all easy to use. Visit http://www.dovepress.com/ testimonials.php to read real quotes from published authors. 